\title{
Las formas de incorporar la orientación a la práctica educativa en la investigación sobre didáctica de la literatura
}

\author{
Ana María Margallo \\ Universitat Autònoma de Barcelona
}

(Texto recibido el 28 de septiembre de 2019; aceptado el 30 de septiembre de 2019; versión final el 30 de septiembre de 2019)

DOI: https://doi.org/10.5565/rev/jt13.854

Resum: Aquest article se centra en les marques que imprimeix a la recerca en didàctica de la literatura l'orientació envers les pràctiques d'ensenyament característica de la disciplina i en les vies que adopta la producció divulgativa per tal de transferir els resultats de la recerca a la comunitat educativa. Utilitza com a referent les produccions de GRETEL, grup de recerca consolidat que, sota la direcció de Teresa Colomer, ha equilibrat la dimensió investigadora i la pràctica al llarg de la seva trajectòria. La caracterització de l'empenta que imprimeix a les recerques el seu ancoratge i projecció envers l'ensenyament s'organitza a partir de dos objectes d'estudi, la literatura infantil i juvenil i la didàctica de la literatura. Els resultats d'aquesta recapitulació mostren els diferents nivells en què s'incorpora la projecció didàctica a les recerques, bé a través de la perspectiva que adopten, bé mitjançant l'elaboració de constructes teòrics o instruments didàctics que donen resposta a les necessitats de l'ensenyament de la literatura. El rastreig de la producció divulgativa il·lustra la diversitat de vies que reforcen l'impacte del resultat de les recerques a la formació docent.

Paraules clau: didàctica de la literatura; investigació; literatura infantil i juvenil; formació docent; transferència

\begin{abstract}
This article focuses on the marks that the orientation towards teaching practices that characterizes the discipline leaves in literature's research in teaching, and on the ways adopted by the informative production to transfer the results of the research to the educational community. To that end, it uses as reference the productions of GRETEL, a consolidated research group that, under the direction of Teresa Colomer, has balanced the dimensions of research and practice throughout its trajectory. The characterization of the footprint left in the research by its anchoring and projection in education is organized in two objects of study: children's and youth literature, and the teaching of literature. The results of this recap show the different levels at which the educational projection is incorporated into research, either through the perspective adopted, or through the elaboration of theoretical constructs or didactic instruments that respond to the teaching needs of the literature. The tracking of informative production illustrates the diversity of ways that reinforce the impact of research results on teacher training.
\end{abstract}

Keywords: literature didactics; research; children's and youth literature; teacher training; transfer 
Resumen: Este artículo se centra en las marcas que deja en la investigación en didáctica de la literatura la orientación hacia las prácticas de enseñanza que caracteriza la disciplina y en las vías que adopta la producción divulgativa para transferir los resultados de la investigación a la comunidad educativa. Para ello, utiliza como referente las producciones de GRETEL, grupo de investigación consolidado que, bajo la dirección de Teresa Colomer, ha equilibrado las dimensiones de la investigación y de la práctica a lo largo de su trayectoria. La caracterización de la huella que deja en las investigaciones su anclaje y proyección en la enseñanza se organiza a partir de dos objetos de estudio, la literatura infantil y juvenil y la didáctica de la literatura. Los resultados de esta recapitulación muestran los distintos niveles en que se incorpora la proyección didáctica a las investigaciones, bien a través de la perspectiva adoptada, bien a través de la elaboración de constructos teóricos o instrumentos didácticos que dan respuesta a las necesidades de enseñanza de la literatura. El rastreo de la producción divulgativa ilustra la diversidad de vías que refuerzan el impacto del resultado de las investigaciones en la formación docente

Palabras clave: didáctica de la literatura; investigación; literatura infantil y juvenil; formación docente; transferencia

El proceso de consolidación de la didáctica de la literatura como disciplina parece haber culminado, una vez superadas las discusiones sobre su especificidad centradas en definir sus fronteras respecto a las disciplinas con las que se relaciona (Bertoni del Guercio, 1992; Campillo, 1990; Lluch, 1998; Mendoza, 2004) y en avalar la legitimidad del corpus literario que constituye uno de sus objetos de estudio (Colomer, 1992, 1998a; Soriano, 1975). La mayoría de edad de la didáctica de la literatura ha ido de la mano de estados de la cuestión sobre los tipos de investigación que la caracterizan (Daunay, 2007; Munita y Margallo, 2019). Este artículo se sitúa en esa misma línea de revisión en tanto se propone caracterizar el impacto que tiene la orientación hacia la práctica educativa en las maneras de investigar en didáctica de la literatura. Puesto que la especificidad del campo va íntimamente asociada a su proyección en la enseñanza, nos parece relevante preguntarnos por las formas en que esa vinculación impregna las líneas de investigación propias de la disciplina.

Nuestra perspectiva sobre las investigaciones en didáctica de la literatura se sitúa en un foco diferente al que acostumbra a adoptarse. En efecto, la necesidad de legitimar el campo centra las revisiones habituales sobre las investigaciones en didáctica de la literatura en las características que avalan su inclusión en la producción científica homologada. Así, en los recopilatorios que muestran los avances de las investigaciones en el marco más amplio de la didáctica de la lengua, se utilizan como hilo conductor los enfoques metodológicos (Camps, 2006; Palou y Guasch, 2013). Las panorámicas resultantes conforman un mapa esclarecedor de las metodologías de investigación que han servido de cauce a las exploraciones en torno a 
los distintos factores involucrados en la didáctica de la lengua y la literatura. Ayudan, así, a la consolidación de estas didácticas específicas como campos del saber que participan de las formas de producción del conocimiento de las ciencias sociales. Pero, una vez reconocida la legitimidad de la didáctica de la literatura para generar investigaciones avaladas científicamente, creemos que tiene interés mostrar la impronta que deja en dichas investigaciones su anclaje en la enseñanza. En la corriente de tensión que sitúa la didáctica de la lengua y la literatura entre la investigación y la intervención, se trata ahora de atender a las marcas que deja la segunda en la primera:

Definirse como una disciplina de intervención habría de reconocer algún impacto en los modos de hacer investigación a partir de la inclusión del trabajo colectivo en relación con las tareas de enseñanza y de formación. Se trataría de un tipo de investigación atenta a las acciones de intervención que desafía a construir otros modos de producción nuevos y originales (Bombini, 2018, p. 7).

Para caracterizar las formas en que la investigación incorpora su proyección hacia la enseñanza, nos ceñiremos a la producción de GRETEL, grupo de investigación de literatura infantil y juvenil y educación literaria de la Universitat Autònoma de Barcelona, en cuya trayectoria se integran las dos vertientes que constituyen el foco de este artículo. El reconocimiento de GRETEL como grupo estable con financiación desde el 2009 y la obtención de proyectos financiados desde el 2003 avalan la producción investigadora del grupo. Su vinculación con las tareas de enseñanza y formación se concreta, tanto en sus numerosas publicaciones divulgativas como en la variedad de iniciativas formativas -Máster en Libros y literatura infantil y juvenil, Máster interuniversitario de Biblioteca escolar y promoción de la lectura, Erasmus Mundus in Children's Literature, Media and Culture- y de transferencia (web http://www.gretel.cat/es/) en las que participa. Por otra parte, resulta oportuno realizar este balance en un momento de transición, con la jubilación de su directora y creadora, Teresa Colomer, estudiosa de referencia que ha contribuido a la consolidación de la didáctica de la literatura en el ámbito hispanoamericano y que ha imprimido al grupo el mismo rigor en sus acciones investigadoras que en las de divulgación y proyección en la enseñanza.

La descripción de las relaciones entre las investigaciones del grupo y sus aportaciones a la dimensión práctica se organiza en torno a los focos que articulan la producción de GRETEL a partir de los elementos del triángulo didáctico (contenidos, docentes y discentes) que constituyen sus objetos de estudio. Se han agrupado estos focos en dos grandes bloques: la 
literatura infantil y juvenil (LIJ) y la didáctica de la literatura. El primero selecciona un tipo de contenidos, el corpus de textos literarios, presente ya desde antes de la formación del grupo en el foco de la tesis de su directora, Teresa Colomer. El segundo reúne las investigaciones sobre los contenidos referidos a los aprendizajes literarios, los docentes y los discentes.

La síntesis sobre las aportaciones del grupo a cada uno de estos campos atenderá a las implicaciones de investigar desde el ámbito de la didáctica. En cada uno mostraremos las marcas que deja en la investigación su anclaje y proyección en la enseñanza de la literatura, así como los tipos de publicaciones y productos dedicados a la transferencia. En este sentido, el artículo aspira a ofrecer una primera caracterización de la especificidad investigadora de la didáctica de la literatura. Al mismo tiempo, el rastreo de la producción divulgativa ligada a las investigaciones constituirá un catálogo de las aportaciones del grupo a la formación docente.

\section{La dimensión formativa de la investigación en LIJ}

Una de las señas de identidad de GRETEL es la mirada sobre la literatura infantil y juvenil que interesa, no como objeto de estudio independiente, como en los enfoques del campo filológico, sino por su aportación a la formación lectora de los niños y jóvenes a los que se dirige. La configuración de ese enfoque investigador se remonta a la publicación basada en la tesis de Teresa Colomer (1998a) donde se propone un acercamiento a las obras literarias que relaciona el análisis de los elementos narrativos con las experiencias y conocimientos que requieren del lector. Este modelo original, fuertemente marcado por la preocupación por las competencias lectoras convocadas por las obras, supone un aporte a la investigación de la LIJ que ha ido consolidándose con otros estudios del grupo. Las tesis de Correro (2018) y Fernández de Gamboa (2018) han aplicado el modelo de análisis a un corpus actualizado de LIJ. Este enfoque de investigación literario-formativo se ha mostrado muy fructífero para aproximarse a subgéneros actuales de la LIJ, en los que resulta relevante relacionar sus características con los retos interpretativos que proponen al lector. En esa línea, las investigaciones sobre el álbum metaficcional (Silva-Díaz, 2005), los álbumes que reformulan el folklore (Bellorín, 2015), la ficción digital (Turrión, 2014; Ramada, 2017) o la poesía infantil (Munita, 2019), proporcionan una comprensión de las nuevas tendencias en LIJ que tiene en cuenta sus aportaciones a la formación literaria de los lectores infantiles y juveniles.

El potencial formativo de la LIJ que han ido relevando las investigaciones del grupo exigía un salto hacia los circuitos de difusión que lo acercase a los mediadores de lectura literaria. Entre las aplicaciones más directas de los estudios sobre LIJ a la formación docente 
destacan las publicaciones sobre los criterios de selección de las lecturas en los distintos niveles de enseñanza (Real y Correro, 2018; Margallo, 2012a) y sobre las orientaciones para integrar sus posibilidades de formación lectora en la mediación (Manresa y Silva Díaz, 2005; Ramada, 2018). A nivel grupal, GRETEL ha consolidado canales de comunicación dirigidos a la formación docente en los que se actualizan las implicaciones didácticas de las investigaciones sobre LIJ:

- La sección de Recomendados, una de las más visitadas de la web de GRETEL, ha renovado el género de la reseña, que acostumbra a centrarse en el resumen argumental o en la información contextual o literaria sobre la obra. La relación que en las reseñas se establece entre la valoración de los aspectos literarios relevantes en cada obra con su aportación a la educación literaria de los lectores infantiles y juveniles, las convierte en un recurso muy útil para la planificación de mediaciones lectoras ajustadas a la funcionalidad formativa de las obras. Las aportaciones mensuales sobre las publicaciones de LIJ (http://www.gretel.cat/es/recomendaciones-lij/) y las que recogen las novedades de ficción digital (http://www.gretel.cat/es/recomendaciones-lij-digital/) constituyen un excelente banco de recursos para los mediadores de lectura literaria, que encuentran aquí una selección de obras elaborada con criterios de calidad así como información sobre sus aportaciones a la creación literaria y a la formación de lectores.

- Las publicaciones colectivas que han cristalizado la doble mirada literaria y formativa sobre las obras en: i) modelos de acercamiento a la comprensión de las competencias lectoras que se asocian a los usos de los recursos narrativos más extendidos en la literatura infantil (Colomer, 2002); ii) panorámicas sobre la producción de literatura juvenil que integran la valoración de sus aportaciones a la formación de lectores literarios (Colomer, 2009). La primera, Siete llaves para valorar las historias infantiles, que se organiza a partir de los elementos narrativos que conforman el itinerario de aprendizaje de los lectores infantiles, modela el análisis literario formativo que los docentes y mediadores de lectura deberían practicar. La segunda, Lecturas adolescentes, analiza la ficción dirigida a esta edad sin perder de vista su aportación a la formación literaria de los adolescentes.

- Un curso online sobre los criterios de selección de lecturas elaborado por GRETEL por encargo del Institut Obert de Catalunya. El formato de miniop permite apropiarse de manera autónoma de contenidos en abierto sobre las claves para seleccionar y valo- 
rar libros para niños y jóvenes. El curso, "Triar llibres per a infants i joves” (Manresa y Bujosa, 2015) se dirige a un público amplio de posibles interesados en el libro infantil y juvenil.

- El Máster Internacional en Libros y Literatura Infantil y Juvenil puede considerarse el colofón de las producciones de transferencia en el que confluyen las aportaciones de todas las investigaciones del grupo, pero especialmente las que tienen que ver con el análisis de la LIJ desde una perspectiva formativa. Este curso on-line, que alcanzó doce ediciones, reúne a profesionales y organizaciones de prestigio del mundo del libro y tiene como punto de partida "la reflexión sobre cómo se las arreglan los libros infantiles para tener lectores; (...) el descubrimiento del modo en que los libros (o la literatura oral) ofrecen a los chicos y a las chicas tanto una idea empírica de lo que se puede esperar de la literatura, como del armazón de desarrollo de las competencias que se precisan para acceder a ella" (Colomer, 2019, p. 41).

Esta recapitulación sobre las formas en las que GRETEL ha integrado la vertiente formativa en sus investigaciones sobre la LIJ permite constatar sus efectos renovadores en la adopción de una perspectiva investigadora que tiene en cuenta al lector en su acercamiento al corpus literario. Por otra parte, las diferentes formas que ha adoptado la comunicación de los resultados de dichas investigaciones deja constancia del esfuerzo divulgativo que debería incorporar todo grupo de investigación que se sitúe en el campo de las didácticas específicas y abre caminos a la adopción de formas diversificadas de transferencia eficaces para tener impacto en la comunidad educativa.

\section{La dimensión programática de las investigaciones en didáctica de la litera- tura}

La trayectoria de GRETEL en tanto grupo que surge en paralelo a la consolidación de la didáctica de la literatura como disciplina en nuestro país, resulta ilustrativa de la proyección de los resultados de las investigaciones en la construcción teórica del paradigma de la educación literaria en torno al que se articula este ámbito del saber. Si bien en cualquier disciplina la relación constante entre la teoría y la práctica investigadora contribuye a la ampliación progresiva de un corpus de conocimientos sobre cada campo del saber, en el caso de la didáctica de la literatura el proceso de conceptualización ha sido muy productivo. Normalmente, este proceso es muy lento y el corpus de conocimientos crece a partir de la suma de aportaciones 
circunscritas al estudio de realidades delimitadas. En cambio, al hacer un balance del resultado de las investigaciones de GRETEL se constata cómo la comprensión del sistema de enseñanza -a partir de investigaciones en torno a los elementos del triángulo didáctico formado por contenidos, docentes y discentes- ha alimentado la elaboración de constructos teóricos con un alto grado de densidad programática. En la aceleración de ese proceso ha sido determinante el carácter de intervención de la disciplina, que debe dar respuesta a las necesidades de enseñanza que surgen de su articulación en torno al nuevo paradigma de educación literaria.

Por otra parte, la fertilidad de la retroalimentación entre la vertiente investigadora y su proyección en la enseñanza produce, además de los constructos teóricos a los que nos hemos referido, instrumentos metodológicos y didácticos. Para ordenar este apartado, por tanto, partiremos de estas producciones, marcando su relación con los objetos de estudio de los que provienen y con las publicaciones en que se divulgan.

Destacaremos en primer lugar los constructos teóricos de GRETEL que dan respuesta a la necesidad escolar de traducir en modelos de programación el nuevo paradigma de la educación literaria. La aplicación de la educación literaria en las aulas, al colocar en el centro de la enseñanza la formación de lectores, frente a los anteriores que pivotaban sobre los contenidos referidos a los autores y las obras desde una perspectiva histórica y formal, precisaba de orientaciones sobre las mejores formas de proceder. La formulación de Teresa Colomer (2005, pp. 84-98) de las líneas de progreso de la competencia literaria, constituye una aportación fundamental que da respuesta a esta necesidad al concretar en qué aspectos debe evolucionar un lector para progresar como tal. Esta delimitación de los objetivos de la formación de lectores culmina un proceso de conceptualización en torno a los componentes de la educación literaria mediante el que Colomer atendió, tanto a la delimitación teórica del paradigma (Colomer, 1996), como a la concreción de las formas de programación escolar más adecuadas para trasladarlo a las aulas. La preocupación de Colomer porque la justificación teórica del modelo de educación literaria fuese de la mano de su proyección en la enseñanza es patente en su apuesta por las secuencias didácticas en forma de proyecto como forma de programación de los aprendizajes literarios acorde con la formación de lectores competentes. Sus numerosas contribuciones al desarrollo de secuencias didácticas literarias (Colomer, 1994, 1998b; Colomer, Milian, Ribas, Guasch y Camps 2003; Colomer, Ribas y Utset, 2003), se enmarcan en la línea de trabajo del Departament de Didàctica de la Llengua i la Literatura de la Universitat Autònoma de Barcelona, que mostró la potencialidad del modelo definido por 
Anna Camps (1994) para integrar el aprendizaje de contenidos en la adquisición de las competencias comunicativas. La consolidación del grupo de académicos vinculados a este Departament como especialistas en didáctica de la lengua y la literatura, va muy ligada a la producción colectiva de propuestas de secuencias didácticas dirigidas al aula explicadas en forma de artículo (Camps, 2003) o desarrolladas como materiales de aula en libros de texto (Camps, 1993). Contemplar la naturalidad de la conexión que, en los inicios de los departamentos de didácticas específicas, se estableció entre los constructos teóricos y las propuestas de programación que los traducían en modelos aplicables al aula produce cierta nostalgia. Resulta evidente, en efecto, que la implantación de los métodos de evaluación de la actividad académica universitaria que se centran exclusivamente en las producciones de investigación ha quebrado la continuidad entre la teoría y la práctica. Una de las consecuencias más dramáticas de la exclusividad de imposición de criterios de valoración ajenos a la naturaleza de las didácticas es la amenaza que suponen para la continuidad de la producción divulgativa y de propuestas programáticas que den sentido a esas investigaciones al desarrollar sus implicaciones didácticas.

En el camino hacia la construcción del constructo teórico de las líneas de progreso, Teresa Colomer (1996) también teoriza sobre las diferentes vías de acceso a la lectura literaria que acabarían concretándose en los espacios de lectura escolar (Colomer, 2005) y en las modalidades de lectura libre y autónoma frente a la guiada e interpretativa (Manresa, 2007; Margallo, 2012b). Todas estas aportaciones confluyen en el constructo teórico de GRETEL sobre los objetivos, modalidades y espacios de la lectura literaria en la escuela que, por su potencialidad como instrumento para la programación escolar de la educación literaria, tiene un gran valor formativo en la formación docente. Aporta, además, una respuesta clara a una de las polémicas más enconadas sobre la enseñanza de la literatura y con más resonancia social: la que cuestiona el mantenimiento de las lecturas obligatorias en la escuela abogando únicamente por la lectura placentera. La certera intervención en el debate de Manresa (2017), en un medio con amplia difusión en los foros docentes, muestra la complementariedad entre las dos modalidades de lectura, guiada y autónoma, y constituye un excelente ejemplo de cómo los constructos teóricos de la didáctica de la literatura aportan pautas de actuación fundamentadas que inciden sobre las cuestiones de fondo que han suscitado debates en torno a la enseñanza de la literatura ${ }^{1}$.

También en torno a la proyección de este constructo en propuestas concretas aplicables a las aulas se despliega parecida actividad a la que se describió en torno a las secuencias 
didácticas. La modalidad de lectura guiada, que se asocia a actividades con una tradición escolar asentada, precisaba de modelos que la renovaran metodológicamente para orientarla a la formación de lectores capaces, depurándola así de la carga erudita o excesivamente formalista que la lastraba y que relegaba al alumno a un papel pasivo en la construcción de significados en torno a las obras. Así, siempre consciente de la necesidad de ofrecer concreciones de los modelos teóricos trasladables al aula, Colomer ${ }^{2}$ colaboró en la colección de Nómades del temps, dedicada a clásicos juveniles, con análisis de las obras afinados y que tienen en cuenta sus aportaciones a la formación literaria de los jóvenes lectores. La renovación metodológica de los dispositivos de ayuda a la comprensión del texto ha tenido continuidad en el proyecto editorial coordinado por Reyes López (2014) en el que, a partir del modelo de Cairney (1992), se concretan guías de lectura sobre obras del catálogo de la editorial Cruïlla que proporcionan al docente: i) una selección de los aspectos literarios sobre los que pivota la construcción de significado de cada obra y que resultan significativos para su comprensión global, y ii) las actividades de explotación didáctica que acompañan al alumno en la construcción y apropiación del sentido de las obras. La modalidad de lectura autónoma, ligada al objetivo de fomento de la lectura, con menor tradición escolar, también ha recibido la atención de GRETEL con propuestas dirigidas a la educación infantil (Colomer y Duran, 2000), primaria (Reyes López, 2011) y secundaria (Margallo, 2012a). En ellas se ofrecen a los docentes líneas de intervención para organizar el tiempo, seleccionar las obras y dinamizar los espacios dedicados a la lectura autónoma en la escuela, de manera que reproduzcan las condiciones que inspiran las prácticas sociales en torno a la lectura.

Otro constructo teórico que supone un avance sustantivo en la concreción de los componentes programáticos de la educación literaria es la propuesta de las dimensiones de la educación literaria de Fittipaldi (2013, pp. 393-442). Las conclusiones de su estudio, que cruza los resultados de la comparación de los aprendizajes literarios presentes en los currículums de diferentes países y las aportaciones de la didáctica de la literatura sobre los aprendizajes que conforman la educación literaria, se orientan a contestar la pregunta de qué deben saber los niños sobre literatura. Fittipaldi propone diez dimensiones definidas a partir de los saberes o aprendizajes literarios que implican, los procedimientos y actitudes con que se relacionan y las prácticas más efectivas para trabajarlos. La potencialidad del constructo de las dimensiones de la educación literaria ha dado sus primeros frutos en el terreno de la investigación y en el de la enseñanza. En el primero, el estudio de Ramada (2018) superpone las dimensiones de Fittipaldi a las características de la ficción digital, lo que le permite armar una propuesta fun- 
damentada de las posibilidades formativas de este tipo de literatura. Se enriquece así la perspectiva formativa de los estudios sobre LIJ, que amplía la mirada sobre las competencias que los textos le piden al lector con las categorías más concretas sobre los tipos de saberes, identificables escolarmente, que permiten al lector infantil y juvenil adquirirlas. En el terreno de la enseñanza, las dimensiones de Fittipaldi constituyen el eje organizador de la más reciente publicación grupal (Colomer, Manresa, Ramada Prieto y Reyes López, 2018) dirigida a los docentes de educación infantil y primaria. Los capítulos de esta obra desgranan los saberes y las actividades que se asocian a cada dimensión con ejemplos de su funcionamiento en las narrativas literarias en estas etapas educativas.

Un segundo grupo de constructos teóricos relevantes son los que proceden de las investigaciones sobre el lector. Este objeto de estudio, muy presente ya en los otros focos tal como se constató al caracterizar la mirada con que se abordan los trabajos sobre LIJ, ha sido el principal eje en las investigaciones sobre respuestas lectoras y sobre hábitos lectores. Las investigaciones de GRETEL sobre las respuestas lectoras de los estudiantes en situaciones de elaboración colectiva del significado de álbumes ilustrados a través de discusiones literarias se insertaron en un proyecto internacional, Visual Journeys, y produjeron una propuesta de categorización de las respuestas lectoras (Fittipaldi, 2012) que permite comprender mejor las claves que utilizan los jóvenes lectores para dar sentido a textos e imágenes de tema migratorio. La utilización de estas categorías en las investigaciones de otros contextos ha permitido ampliar el modelo con los tipos de respuesta que utilizan los alumnos en discusiones que se llevan a cabo en un aula de primaria de manera sostenida así como ver su evolución en el tiempo (Reyes López, 2015) o explorar las respuestas que genera la lectura de narraciones digitales en el contexto familiar (Aliagas y Margallo, 2016). La proyección didáctica de las investigaciones sobre respuestas lectoras ha tenido su aplicación más inmediata en la tipificación de las funciones de las intervenciones del docente en la discusión literaria en relación al tipo de respuesta que generan (Munita y Manresa, 2012). La aportación de Munita y Manresa resulta muy necesaria en tanto amplía la propuesta de Chambers (2007), que sugiere tipos de preguntas, genéricas y focalizadas sobre las características de los textos, adecuadas para suscitar el debate interpretativo. La contribución de los miembros de GRETEL supone un paso más porque tiene en cuenta cómo conducir la discusión en función de los tipos de respuesta que se quiere generar para que los lectores progresen en la construcción de significados sobre las obras discutidas. 
Otro constructo referido al lector pero basado en una investigación de corte sociológico, es la propuesta de Manresa (2009) para caracterizar el hábito de lectura. Su estudio longitudinal sobre lo que leían y expresaban sobre las lecturas ochenta adolescentes durante tres cursos escolares, precisó la construcción de unas variables que le permitieran incorporar al análisis de la evolución de los hábitos de lectura de esos estudiantes los resultados sobre la cantidad de libros leídos, su variedad y la capacidad de valorarlos. Esta reformulación de las dimensiones que componen el hábito de lectura, además de proponer un modelo de análisis más comprensivo para las investigaciones sobre hábitos lectores, lleva implícito un replanteamiento de los objetivos escolares en torno a la formación de lectores que permitan incidir sobre las tres variables: cantidad de lecturas, variedad de las obras leídas y tipo de discurso que el lector construye sobre ellas. En el libro que difunde los resultados de la tesis, Manresa (2013) desglosa la variedad de componentes que conforman los hábitos lectores de los adolescentes, y despliega una propuesta sobre las intervenciones que resultan más adecuadas para incidir sobre cada uno de ellos según los distintos perfiles lectores. La producción divulgativa se completa con un curso telemático, enmarcado en las iniciativas para impulsar la lectura del Departament d'Ensenyament de la Generalitat de Catalunya, que proporciona pautas a los docentes de todas las etapas para incidir sobre los hábitos lectores de los distintos perfiles de alumnos desde la biblioteca escolar (Manresa, 2012).

Para terminar, nos detendremos en la proyección en la enseñanza que han tenido las investigaciones de Munita sobre los docentes $(2014,2016)$. Su concepto de "ecosistema mediador" proporciona una clave muy adecuada para entender la influencia de los contextos institucionales que conforman el entorno escolar en el desarrollo de las trayectorias profesionales de los docentes. Se trata de un constructo que amplía la comprensión sobre los elementos que conforman el rol de mediador literario al incorporar tanto las interrelaciones entre los docentes y la institución escolar como las acciones que se movilizan en cada sistema pedagógico en relación a las prácticas relacionadas con la lectura literaria. La potencialidad del constructo del ecosistema mediador para la formación docente está en proceso de incorporación al diseño de proyectos de formación docente. Resulta especialmente atractivo para entender mejor los factores que determinan la cristalización del rol de mediador de lectura literaria en los cursos de formación de centro, y para proponer acciones encaminadas a incidir sobre dichos factores.

De la tesis de Munita (2014) emerge también un instrumento metodológico, la pauta de sistematización de Prácticas Didácticas en Educación Lectora y Literaria (PRADEL), que se diseñó para observar las prácticas docentes en este ámbito. La confección de dicho instru- 
mento supuso un enorme esfuerzo de síntesis de los aportes de la didáctica de la literatura que sirven a la formulación de los criterios que permiten caracterizar las actuaciones docentes esenciales para aplicar los principios de la educación literaria al aula. La difusión, que se presume inmediata, de esta herramienta tan útil para la formación dotará a los docentes de una pauta tanto para la planificación de clases que encajen en el modelo de educación literaria como para la regulación de sus prácticas en relación a la lectura literaria.

Además de las producciones divulgativas ligadas a los constructos e instrumentos que emergen de las investigaciones sobre didáctica de la literatura, el Máster de Biblioteca escolar y promoción de la lectura constituye una plataforma en la que estos constructos e instrumentos se integran en los cursos dirigidos al profesorado de primaria y secundaria, y a los profesionales de la promoción lectora y del mundo editorial a los que ofrece formación sobre el funcionamiento de la biblioteca escolar y los proyectos sobre LIJ que inciden en los hábitos lectores.

\section{A modo de cierre}

La panorámica que hemos trazado sobre las conexiones entre la dimensión investigadora y su proyección en la enseñanza en la trayectoria de GRETEL confirma una línea de evolución paralela a la que señala Camps (2017) para las investigaciones en didáctica de la lengua a partir de los '80: responder al impulso por mejorar la enseñanza de la lectura y la escritura en el marco de la renovación metodológica que articula los currículums en torno a las competencias. Las investigaciones de GRETEL, que se pueden considerar representativas del ámbito de la didáctica de la literatura, responden a esta misma inquietud por atender a las necesidades de renovación que aquí se articulan en torno a la educación literaria de lectores competentes.

Este compromiso con la renovación de la enseñanza de la literatura para que integre los principios de la educación literaria es un motor muy potente en la trayectoria de GRETEL que determina, tanto las características de sus investigaciones, como las relaciones que éstas establecen con las producciones divulgativas que apuntan a la transferencia de los resultados de las investigaciones al mundo de la enseñanza. La manera en que las investigaciones de GRETEL han incorporado la proyección didáctica ha mantenido algunas constantes:

- Incorporación de la dimensión formativa a la perspectiva de investigación que se realiza en diferentes niveles. Así, en las investigaciones sobre LIJ, se imbrica el análisis de las características del corpus estudiado con el de sus aportaciones a la formación de los lectores, en una perspectiva novedosa que integra al lector en la mirada sobre el 
corpus literario. En las de didáctica de la literatura, la proyección en la enseñanza se traduce en las implicaciones didácticas de los resultados de la investigación o en la emergencia de constructos teóricos e instrumentos que surgen durante el proceso investigativo y que constituyen una aportación a la enseñanza. Ha resultado relevante la proyección programática de los constructos teóricos que, en el seno de investigaciones sobre el triángulo didáctico, constituyen un aporte a la definición del paradigma de la educación literaria y a su concreción en el aula.

- La fuerte interrelación entre los aportes procedentes de la investigación y los que se orientan a la enseñanza imprime a la trayectoria del grupo una gran coherencia en tanto se superponen de manera significativa las aportaciones de ambos campos. Podría decirse que el compromiso con la dimensión práctica actúa como un elemento cohesionador que vela porque los objetivos de los proyectos de investigación den respuesta a las necesidades de enseñanza.

El registro de las producciones divulgativas que se han ocupado de trasladar los resultados de las investigaciones a la formación docente ha permitido constatar:

- La diversidad de modalidades discursivas y de canales utilizados para la divulgación y la transferencia. El gran número de artículos en revistas dirigidas a los docentes, se complementa con las publicaciones colectivas que jalonan la trayectoria del grupo y proyectan las implicaciones didácticas de los avances ligados a proyectos de investigación. También se han explorado vías diversas para la formación docente, que contemplan tanto la publicación de materiales de aula (en forma de libros de texto o de guías de lectura) como otras menos convencionales como los cursos en línea que se ofrecen en colaboración con otras instituciones (IOC, Departament d'Ensenyament). Además de estas producciones puntuales, GRETEL mantiene una oferta formativa de postgrado y una página web que se alimentan y actualizan con la producción científica del grupo.

- El acompasamiento de las producciones de investigación y las de divulgación permite que se avance de manera paralela en ambos campos, para que la traducción de los resultados de la investigación en orientaciones para el aula no quede relegada a un segundo término.

Estas aportaciones dejan constancia del esfuerzo sostenido de GRETEL por atender a la producción divulgativa, aun cuando el sistema actual de promoción académica no lo facili- 
ta. Aun cuando los criterios de valoración de grupos e investigadores universitarios se centran en la producción científica publicada en revistas del circuito universitario, GRETEL ha sabido conciliar la dimensión investigadora y la didáctica. Y, como se desprende de la trayectoria que ha trazado el presente artículo, esta conexión está muy ligada a la naturaleza de un grupo que investiga sobre didáctica de la literatura y determina el tipo de investigación que se realiza. Es de esperar que las didácticas específicas vayan encontrando acomodo en el sistema de reconocimiento académico, y que las aportaciones de grupos como GRETEL a la divulgación de sus investigaciones no sólo obtengan reconocimiento sino que ayuden a conformar las reglas de un sistema evaluador sensible a la imprescindible conexión que en estas disciplinas se da entre la dimensión investigadora y su proyección en la enseñanza.

\section{Referencias}

Aliagas, C., y Margallo, A.M. (2016). Children's responses to the interactivity of storybook apps in family shared Reading events involving the iPad. Literacy, 51 (1), 44-52. DOI: https://doi.org/10.1111/lit.12089

Bellorín, B. (2015). De lo universal a lo global: nuevas formas del folklore en los álbumes para niños (Tesis doctoral). Universitat Autònoma de Barcelona. Recuperado de http://hdl.handle.net/10803/311617

Bertoni del Guercio, G. (1992). L'ensenyament del text literari. En T. Colomer (coord.), Ajudar a llegir. La formació lectora a primària i a secundària (pp. 87-104). Barcelona: Barcanova.

Bombini, G. (2018). Didáctica de la lengua y la literatura: entre la intervención y la investigación. Bellaterra Journal of Teaching \& Learning Language \& Literature, 11 (4), 5-20. DOI: https://doi.org/10.5565/rev/jt13.784

Campillo, M. (1990). Text/context: algunes consideracions sobre l'ensenyament de la literatura. En A. Camps (coord), Text $i$ ensenyament. Una aproximació interdisciplinària (pp. 95-117). Barcelona: Barcanova.

Cairney, T. H. (1992). Enseñanza de la comprensión lectora. Madrid: Morata.

Camps, A. (1993). Ull de bou. Llengua: Educació primària, cicle mitjà. Barcelona: Barcanova.

Camps, A. (1994). Projectes de llengua entre la teoria i la pràctica. Articles de Didàctica de la llengua i la literatura, 2, 7-20. 
Camps, A (comp.) (2003). Secuencias didácticas para aprender a escribir. Barcelona: Graó.

Camps, A. (coord.) (2006). Diálogo e investigación en las aulas. Investigaciones en didáctica de la lengua. Barcelona: Graó.

Camps, A. (2017). 25 anys de recerca en didàctica de la llengua. Bellaterra Journal of Teaching \& Learning Language \& Literature, 10 (1), 9-19. DOI: http://dx.doi.org/10.5565/rev/jt13.721

Chambers, A. (2007). Dime. Los niños, la lectura y la conversación. México D.F.: Fondo de Cultura Económica.

Colomer, T. (1992). Una nova literatura per als nous lectors. En T. Colomer (coord), Ajudar a llegir. La formació lectora a primària i a secundària (pp.105-127). Barcelona: Barcanova

Colomer, T. (1994). La lectura en els projectes de treball. Articles de Didàctica de la llengua $i$ la literaturas, 2, 63-71. (Traducción al castellano en Colomer, T. (2001). Enseñanza de la literatura y proyectos de trabajo. Lulú Coquette. Revista de didáctica de la lengua y la literatura, 1, 99-111.

Colomer, T. (1996). La didáctica de la literatura: temas y líneas de innovación. En C. Lomas (coord.), La educación lingüistica y literaria en la enseñanza secundaria (pp. 123142) Barcelona: ICE Universitat de Barcelona-Horsori.

Colomer, T. (1998a). La formació del lector literari. Barcelona: Barcanova.

Colomer, T. (1998b). Itineraris literaris: la poesia com a expresió de sentiments. En A. Camps y T. Colomer (coords), L'ensenyamant i l'aprenentatge de la llengua i la literatura en l'educació secundària (pp.177-188). Barcelona: ICE/Horsori.

Colomer, T. (dir) (2002). Siete llaves para valorar las historias infantiles. Madrid: Fundación Germán Sánchez Ruipérez.

Colomer, T. (2005). Andar entre libros. México D.F.: Fondo de Cultura Económica.

Colomer, T. (coord.) (2009). Lecturas adolescentes. Barcelona: Graó.

Colomer, T. (2019). La xarxa d'un postgrau: el màster de llibres i literatura per a infants i joves. / La red de un postgrado: el máster de libros y literatura para niños y jóvenes. Ooohéee: estudis sobre la creació $i$ edició infantil i juvenil, 4, 32-52.

Colomer, T., y Duran, T. (2000). La literatura en la etapa infantil. En M. Correig y M. Bigas (coords.), Didáctica de la lengua en la educación infantil (pp. 213-250). Madrid: Síntesis. 
Colomer, T., Milian, M., Ribas, T., Guasch, O., y Camps, A. (2003). El héroe medieval: un proyecto de literatura europea. En A. Camps (comp.), Secuencias didácticas para aprender a escribir (pp. 71-82). Barcelona: Graó.

Colomer, T., Ribas, T., y Utset, M. (2003). La escritura por proyectos: Tú eres el autor. En A. Camps (comp.), Secuencias didácticas para aprender a escribir (pp. 61-70). Barcelona: Graó.

Colomer, T., Manresa, M., Ramada Prieto, L., y Reyes López, L (2018). Narrativas literarias en educación infantil y primaria. Madrid: Síntesis.

Correro, C. (2018). Els llibres infantils al segle XXI (Tesis doctoral). Universitat Autònoma de Barcelona. Recuperado de http://hdl.handle.net/10803/665173

Daunay, B. (2007). État des recherches en didactique de la littérature. Revue Française de Pédagogie, 159, 139-189.

Fernández de Gamboa, K. (2018). A través del nuevo milenio y lo que el lector literario encontró allí: la narrativa infantil para lectores de 8 a 10 años. (Tesis doctoral) Universitat Autònoma de Barcelona. Recuperado de http://hdl.handle.net/10803/665731

Fittipaldi, M. (2012). La categorización de las respuestas infantiles ante los textos literarios. Análisis de algunos modelos y propuestas de clasificación. En T. Colomer y M. Fittipaldi (coords.), La literatura que acoge: Inmigración y lectura de álbumes (pp. 6986). Caracas, Barcelona: Banco del Libro-GRETEL.

Fittipaldi, M. (2013). ¿Qué han de saber los niños sobre literatura? Conocimientos literarios y tipos de actuaciones que permiten progresar en la competencia literaria (Tesis doctoral). Universitat Autònoma de Barcelona. Recuperado de https://hdl.handle.net/10803/131306

Lluch, G (1998). El lector model en la narrativa per a infants i joves. Valencia: Servei de Publicacions Universitat de València.

Manresa, M. (2007). Lectures dels adolescents: entre la tria personal i la selecció escolar. Articles de Didàctica de la Llengua i la Literatura, 41, 71-86.

Manresa, M. (2009). Els hàbits lectors dels adolescents. Efectes de les actuacions escolars en les pràctiques de lectura (Tesis doctoral). Universitat Autònoma de Barcelona.

Barcelona. Recuperado de http://hdl.handle.net/10803/4685

Manresa, M. (coord.) (2012). Biblioteca escolar i gust per llegir. Barcelona: Generalitat de Catalunya. Recuperado de http://ateneu.xtec.cat/wikiform/wikiexport/cmd/bib/begl/index 
Manresa, M. (2013). L'univers lector adolescent. Dels hàbits de lectura a la intervenció educativa. Barcelona: Associació de Mestres Rosa Sensat.

Manresa, M (2017) Lectures obligatòries, sí o no? El diari d'educació. Blog de l'associació de mestres Rosa Sensat. 10/01/2017. Recuperado de http://diarieducacio.cat/blogs/rosasensat/2017/01/10/lectures-obligatories-si-o-no/

Manresa, M., y Silva Díaz, M. C. (2005). Dialogar per aprendre literatura. Articles de didàctica de la llengua i la literatura, 37, 45-56.

Manresa, M., y Bujosa, C. (coords) (2015). Triar llibres per a infants i joves. Recuperado de http://miniops.ioc.cat/10/

Margallo, A. M. (2012a). El reto de elaborar un corpus para la formación literaria de adolescentes reticentes a la lectura. Anuario AILIJ, 10, 69-85.

Margallo, A. M. (2012b). Claves para formar lectores adolescentes con talento. Leer.es. Recuperado de $\quad$ https://leer.es/recursos/investigar/detalle//asset_publisher/3fAFCQK 7mwkO/content/claves-para-formar-lectores-adolescentescon-talento-ana-mariamargallo;jsessionid=0309CE74E41723B75AA50742FD817E53

Mendoza, A. (2004). La educación literaria: bases para la formación de la competencia lecto-literaria. Málaga: Aljibe.

Munita, F. (2014). El mediador escolar de lectura literaria. Un estudio del espacio de encuentro entre prácticas didácticas, sistemas de creencias y trayectorias personales de lectura (Tesis doctoral). Universitat Autònoma de Barcelona. Recuperado de http://hdl.handle.net/10803/313451

Munita, F. (2016). Prácticas, creencias y hábitos lectores del profesor en una escuela exitosa en la promoción lectora. Ocnos, 15 (2), 77-97. DOI: https://doi.org/10.18239/ocnos_2016.15.2.1140

Munita, F. (2019). Hacia una "poesía para niños también”. Tendencias de la poesía infantil en dos premios del ámbito hispanoamericano (2004-2017). En A. Córdova (coord.), Renovar el asombro: Un panorama de la poesía infantil y juvenil contemporánea en español (pp. 103-141). Cuenca: Ediciones de la Universidad de Castilla-La Mancha.

Munita, F., y Manresa, M. (2012). La mediación en la discusión literaria. En T. Colomer y M. Fittipaldi (coords.), La literatura que acoge (pp. 119-143). Barcelona: Gretel-Banco del Libro. 
Munita, F., y Margallo, A.M. (2019). La didáctica de la literatura: configuración de la disciplina y tendencias de investigación en el ámbito hispanohablante. Perfiles Educativos, 41 (164), 154-170. DOI: http://dx.doi.org/10.22201/iisue.24486167e.2019.164.58825

Palou, J. y Guasch, O. (coords.) (2013). Monográfico: El estudio de la interacción en el aprendizaje de la lengua y la literatura: retos metodológicos. Cultura \& Educación, 25 (4).

Ramada, L. (2017). Esto no va de libros. Literatura infantil y juvenil digital y educación literaria (Tesis doctoral). Universitat Autònoma de Barcelona. Recuperado de http://hdl.handle.net/10803/460770

Ramada Prieto, L. (2018). “Cariño, tenemos que hablar”. Ficción digital infantil y juvenil y su mediación. Revista Peonza, 126, 7-15.

Real, N., y Correro, C. (2018). Valorar la literatura infantil digital: propuesta práctica para los mediadores. Revista Textura, 20 (42), 8-33.

Reyes, López. L. (2011). Fer lectors en un context advers. Articles de Didàctica de la Llengua $i$ de la Literatura, 53, 57-67.

Reyes López, L. (2014). Programa Exxit lector 2014. Educació Primària. Disponible en: http://www.cruilla.cat/exit-lector-cicle-superior

Reyes López, L. (2015). La formació literària a primària. Impacte de l'actuació educativa en l'evolució de respostes lectores (Tesis doctoral). Universitat Autònoma de Bacelona. Recuperado de: http://hdl.handle.net/10803/308312

Silva-Díaz, M.C. (2005). Libros que enseñan a leer: álbumes metaficcionales y conocimiento literario (Tesis doctoral). Universitat Autònoma de Barcelona. Recuperado de: http://hdl.handle.net/10803/4667

Soriano, M. (1975). Guide de la littérature enfantine. Paris: Flammarion. (Trad. Cast. La literatura para niños y jóvenes. Guía de exploración de sus grandes temas. Buenos Aires: Ediciones Colihue, 1995).

Turrión, C. (2014). Narrativa infantil y juvenil digital. ¿Qué ofrecen las nuevas formas al lector literario? (Tesis doctoral). Universitat Autònoma de Barcelona. Recuperado de: http://hdl.handle.net/10803/285652

\section{Notas}

${ }^{1}$ La necesidad de definir una postura informada en torno a polémicas con calado social se ha dado en otras ocasiones, como la síntesis que ofrece Colomer en torno a la censura de los libros sexistas:

http://www.gretel.cat/es/noticias/retiramos-los-libros-sexistas/ 
${ }^{2}$ Los Apéndices que reúnen estos análisis de obras como Colmillo Blanco, Dr. Jekyll y Mr. Hyde, El perro de los Baskerville, El retrato de Dorian Gray y otras pueden consultarse en la web de GRETEL:

http://www.gretel.cat/lectures/page/8/

\section{Información sobre la autora:}

Ana María Margallo es profesora en el Departamento de Didáctica de la lengua y la literatura de la Universitat Autònoma de Barcelona. Coordina el grupo GRETEL (Grupo de Investigación en Literatura Infantil y Juvenil y Educación Literaria) reconocido por la Agencia AGAUR de la Generalitat de Catalunya como equipo estable de investigación con financiación (2009SGR1477). Sus líneas de investigación actuales comprenden los usos y recepción de la literatura infantil y juvenil digital, las aportaciones del trabajo por proyectos a la formación del lector literario, y las posibilidades de la investigación-acción para la formación del profesorado.

E-mail: anamaria.margallo@uab.cat

Para citar este artículo:

Margallo, A.M. (2019). Las formas de incorporar la orientación a la práctica educativa en la investigación sobre didáctica de la literatura. Bellaterra Journal of Teaching \& Learning Language \& Literature, 12(3), 7-25. DOI: https://doi.org/10.5565/rev/jtl3.854 